\title{
Experiment on the Heat Fatigue Damage Behavior of Optical Fiber
}

\author{
Zhiyuan $\operatorname{Pan}^{1, \text { a * }}$ \\ ${ }^{1}$ School of science, Wuhan university of technology huaxia college, No. 589, Guansan Road, Wuhan \\ 430070, China \\ apzy820126@163.com
}

Keywords: Fiber optic sensor; Bare fiber; Thermal fatigue; Stiffness; Damage

\begin{abstract}
Fiber-optic sensors are widely used in the long-term health monitoring of major engineering structures, and it will generate heat fatigue damage under the influence of the environment, which greatly affect the stability of the optical fiber sensor. In this paper, experimental analysis for optical properties and thermal fatigue damage were studied. Based on the experimental data, the thermal expansion coefficient of the fiber is approximately $0.88 \times 10^{-6} /{ }^{\circ} \mathrm{C}$. Points out at temperature cycling conditions, the fiber damage occurred, the elastic modulus exhibits decreasing trend. Analysis of the mechanism of fiber thermal fatigue damage and the impact on performance of the optical fiber sensor. The results of this research provide a reference to predict the durability and life of fiber optic sensors.
\end{abstract}

\section{Introduction}

Due to many advantages of optical fiber sensor, such as light weight, durability, and long-term stability, it can be used to monitor the stress and strain in building structures. And for optical fiber Bragg grating sensor, the sensitive to temperature and strain making it widely used in the engineering field. Because the widely used in the long-term health monitoring and diagnosis of large engineering structures, more and more attention has pay to the long-term stability of optical fiber Bragg grating sensor [1]. So far the study of the stability of optical fiber was focused on its mechanical fatigue and the probability of failure after long-term use. During the service time of optical fiber Bragg grating sensor, the alternation temperature and corrosion environment could cause fatigue damage and changed the stiffness (elasticity modulus). These changes would impact the effective refractive index and fiber cycle and lead to reduce the stability of its sensing properties. Duhamel [2] has concluded the thermal stress calculation formula after repeated experimental studies. Pan [3] has interpretation of the concept of thermal fatigue damage by study on thermal fatigue damage of flat glass. This provides a reference for the study on thermal fatigue damage. Nowadays the international studies on thermal fatigue were improve from qualitative to quantitative[3-10]. But all of these researches were used low cycle fatigue to imitate the thermal fatigue. In this paper, we study the thermal fatigue damage property of optical fiber by temperature cycling test on the unconstrained bare optical fiber.

\section{Experiment Method}

References are cited in the text just by square brackets [1]. (If square brackets are not available, slashes may be used instead, e.g. /2/.) Two or more references at a time may be put in one set of brackets $[3,4]$. The references are to be numbered in the order in which they are cited in the text and are to be listed at the end of the contribution under a heading References, see our example below.

Experiment Principle.This experiment calculated the damage variable indirectly by measuring the elastic modulus of bare optical fiber after cycling heat from the macro aspects. The relationship between damage variable $\mathrm{D}$ and effective elastic modulus can be expressed as:

$$
D=\frac{E-\tilde{E}}{E}=1-\frac{\tilde{E}}{E}
$$


Where when $\mathrm{D}$ equal 0 , the specimens were scatheless. When $\mathrm{D}$ equal 1 , the specimens were damaged completely (fracture).When $\mathrm{D}$ was between 0 and 1 , the specimens damaged varying degrees. In general, the deformation caused by thermal expansion and contraction was reversible and the deformation was elastic. After repeated thermal cycling, the irreversible deformation was appeared. This indicating that the optical fiber specimens exist a thermal fatigue phenomena in this temperature range. But no matter how many thermal cycles, there were not unrecoverable deformation exist, then there were no thermal fatigue in this temperature range.

Specimens Preparation. Materials used in this experiment: Glass containers, acetone, alcohol, cotton balls, some optical fiber, 5 to 8 pairs of iron clamp piece, an appropriate area of black thin sponge, iron wire several and 5 to $8200 \mathrm{~g}$ weights. The optical fiber was produced by Wuhan Yangtze Optical Fibre and Cable Joint Stock Limited Company. The chemical composition of outside protective layer was poly aluminum chloride (PAC). The acetone was laboratory dedicated pure acetone which produced by Tianjin Fu Yu Fine Chemical Co., Ltd.

Specimens Preparation. The length of the fiber cut into a number of segments of $1500 \mathrm{~mm}$, then placed in a glass vessel equipped with an acetone solution and soak one hour.Removed the soaked fiber from the container with tweezer, then wipe the fiber along its length with a cotton ball with alcohol.Placed the optical fiber perpendicular to the ground, then shell the coating slowly along the length direction. The bare optical fiber specimens was obtain.Wipe the obtained bare optical fiber with cation ball with alcohol again.Repeat the above steps, preparation five bare fiber specimen.Alignment placed the iron clamping piece with bolt and sponge on both sides of the $1000 \mathrm{~mm}$ long template. Then put the specimens centered on the clamping piece, a piece of same shape sponge was then placed, and the other piece of clamp was pressed against the sponge.Tightening the nut evenly with pliers. Placed the next optical fiber $5 \mathrm{~cm}$ away from the last optical fiber with same method until five optical fibers were placed along the template.The iron wire were wound on both sides of the clip's bolt and around in a circle, which convenient for hang weight on the steel frame.The completed specimens was show in Fig. 1. Both sides is iron clip. The screws outside of the two pieces of iron clamp were tied with appropriate iron wire. In the middle was bare optical fiber sample.

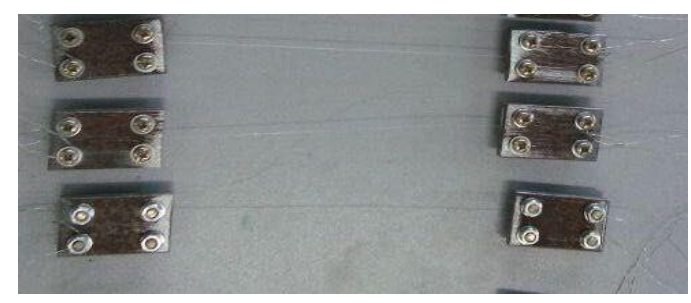

Figure.1 Optical fiber specimens

Experimental Procedure. Main equipment: HZ891 eddy current displacement transducer produced by Shanghai Airlines Vibration Instrument Co., Ltd., the range is $10 \mathrm{~mm}$, with a resolution of $0.1 \mu \mathrm{m}$.UT3202FRS-ICP Data collecting instrument produced by Wuhan Excellent Thai Electronic Technology Co., Ltd.FGH-10A Heater produced by Gree. input / output power of 1000w, power supply $220 \mathrm{~V}, 50 \mathrm{~Hz}$.

Thermocouple Thermometer. Main equipment selection:Because the high accuracy with resolution up to $0.1 \mu \mathrm{m}$ and non-contact, we chose the Eddy current displacement transducer in our experiment. In this study, we get the length changes of the optical fiber by measuring the changes of vertical distance between the bottom of weight and the probe of eddy current displacement transducer. Its mechanism was shown in Fig. 2. 


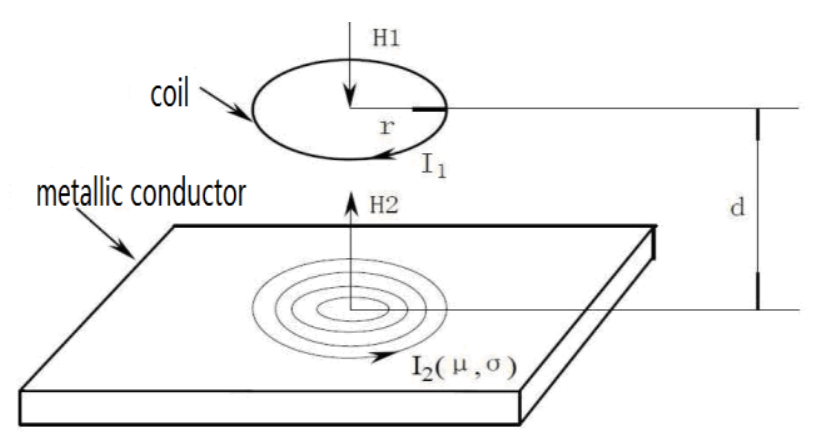

Figure 2. The mechanism of eddy current effect

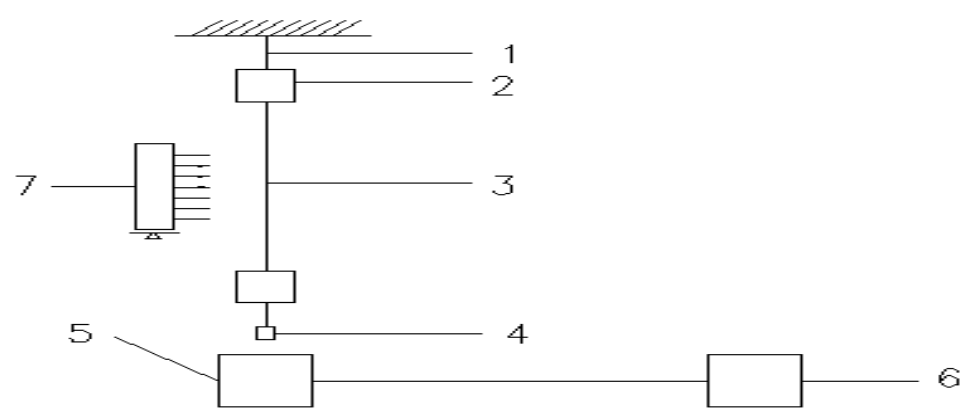

Figure 3. Experimental device

1-Iron wire; 2-Iron clamp piece; 3-Optical fiber specimens; 4-Weight; 5-Eddy current displacement transducer; 6- Data collecting; 7- Heater;

Experiment Procedure.Hang the wire ring which on the side of the specimen on the steel frame of the experimental device. And on the other side, a 200g weight was tied on the wire ring. Then hold the weight slowly decentralization. The whole process should be carefully to avoid stress in optical fiber. The experimental device was shown in Fig. 3. Build the experimental device. The button of the weight parallel to the sensor. The distance between them is $2 \mathrm{~mm}$.Rotate the heater back to the specimen. Then turn on the heater. Measure the temperature with a thermocouple thermometer when the temperature is stable. The temperature changes is $\Delta T=T_{1}-T_{2}$.Remove the heater after heating the specimens two minutes. Then leave the specimens under the room temperature for two minutes. According to this method the temperature recycled every four minutes. Recording the data get from the collecting $L_{\mathrm{i}} 、 L_{\mathrm{j}}$ respectively when suffering 50,100,150, 200, 250, 300,450, 600,750, 900, $1050,1200,1350,1500,1650,1800,1950$ and 2000 cycles of heating. The length of optical fiber changes $\Delta L=L_{\mathrm{j}}$. Calculate the effective elastic modulus according to the experimental data. And then draw the curves of the relationship between thermal cycle times and effective elastic modulus and the curves of the relationship between thermal cycle times and damage variable.

\section{Results and Discussion}

In our experiments measured the heated temperature is $68^{\circ} \mathrm{C}$. The room temperature is $8^{\circ} \mathrm{C}$.So the temperature difference is $\Delta \mathrm{T}=60^{\circ} \mathrm{C}$.

We can preliminary estimated the thermal expansion coefficient by the different length between 50th and 250th cycles of heating. Where $L_{\mathrm{i}}=\alpha \times \Delta T \times L, \Delta \mathrm{T}=60^{\circ} \mathrm{C}, \mathrm{L}=500 \mathrm{~mm}$. The relationship between thermal cycle times and thermal expansion coefficient was shown in Fig. 4. We can get the average thermal expansion coefficient of the bare optical fiber is $\alpha=0.88 \times 10^{-6} /{ }^{\circ} \mathrm{C}$. The horizontal line was the thermal expansion coefficient we preliminary estimated. 




Figure.4 Relation between times of thermal cycle and thermal expansion coefficient

In this experimenter the diameter of the bare optical fiber was $125 \mu \mathrm{m}$. We can calculate correlation dada according to the following formula:

Strain:

$$
\varepsilon=\Delta L / L=\Delta L / 500
$$

Effective elastic modulus:

The original elastic modulus:

$$
\tilde{E}=\sigma / \varepsilon=1.996 \times 10^{10} / \Delta L
$$

Damage variable:

$$
E=7.2 \times 10^{10} \mathrm{~Pa}
$$

$$
D=\frac{E-\tilde{E}}{E}=1-\frac{\tilde{E}}{E}=1-\frac{1.996 \times 10^{10}}{7.2 \times 10^{10} \Delta L}=1-\frac{0.277}{\Delta L}
$$

We can get the relationship between the effective elastic modulus and the number of cycles which shown if Fig. 5 according to the change of the length of the specimen. And the relationship between number of cycles and damage variable was shown in Fig. 6.

As shown in Fig. 5, the effective elastic modulus almost keep constant at the initial 1200 times cycles. And $E=E$.Then the effective elastic modulus decrease gradually as the increasing of the times of thermal cycles.

Because of the damage of the optical fiber is cumulative and irreversible, the experiment could be cumulative until 2000 times thermal cycles.



Figure.5 relationship between times of thermal cycles and effective elastic modulus 


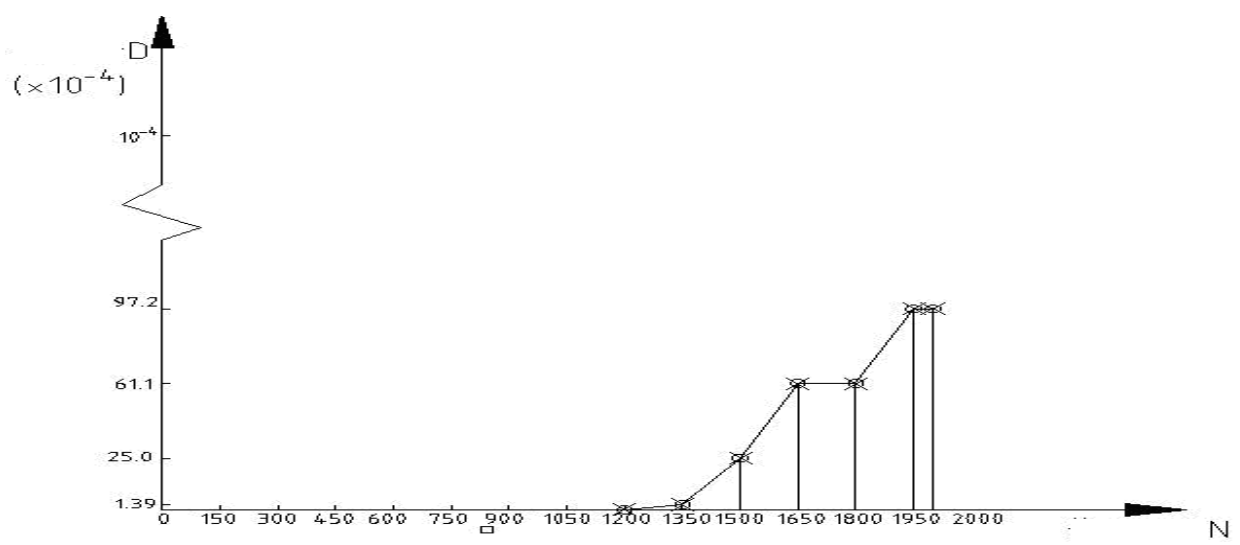

Figure.6 relationship between times of thermal cycles and damage variable

As shown in Fig. 6, there were no damage at the initial 1200 times cycles. $D=0$. Then the damage variable increasing gradually as the increasing of the times of thermal cycles. But the value of the thermal cycles was still small due to the limit of time. The times of thermal cycles was not enough so the damage was not obvious. Through the analysis above, we can infer the optical fiber's thermal fatigue damage mechanism. Within a certain number of thermal cycles, the length of the fiber could be recovery and the damage variable is zero. After reach a certain number of thermal cycles, it may appear within certain crack, hollow or plastic deformation etc. and make the effective elastic modulus decreases. As in the experiment the length of the specimen increased after thermal cycle. This means the optical fiber was damaged varying degrees because the damage is irreversible

\section{Conclusions}

When at initial the optical fiber specimens after a certain number of cyclic heating, its deformation is still belongs to the elastic deformation. There were no thermal fatigue phenomenon exit in this process. After many thermal cycles the optical fiber specimens appeared the unrecoverable deformation which means there were thermal fatigue damage exist in the temperature. The thermal expansion coefficient of the optical fiber is about $0.88 \times 10^{-6} /{ }^{\circ} \mathrm{C}$. After the optical fiber specimen reached a certain number of thermal cycles, the specimen would appear different degrees of damage as the increase of number of thermal cycles.

\section{Acknowledgements}

The work is supported with scientific research plan guide project B2014278, from Hubei province ministry of education.

\section{References}

[1] Liang Lei, Jiang Desheng, Zhou Xuefang, Nan Qiuming. The application of fiber Bragg grating sensing technology in experimental mechanics [J]. Journal of nanchang university, 2003, 25 (2) : 14-18.

[2] Xie Changsheng, Zhao Jiansheng. An Approach to Developing a Hot-work Die Steel for High Temperature Application [J]. Mater Sci. Aug. 1990:A124:1-9.

[3] Pan Suying, Song Xianhui. Thermal fatigue damage of Flat glass [J]. Journal of Wuhan University of Technology. 1993. 15 (2): 37-40.

[4] Liu Tai, Zhai Qingshi, Liu Hongfeng. An analysis of the reliability factors of communication optical fiber [J]. Journal of optical communications research. 2012 (5): 33-36 
[5] Wang Baozhu, Deng Honglin, Li Xiaorui, Zhan Yimin. Optical fiber Life expectancy for guidance fiber [J]. Journal of applied optics. 2005, 26 (6): 41-45

[6] Zhang Xiaoli, Liang Dakai, Lu Jiyun et al..A high reliable optic fiber Bragg grating sensor network design[J]. Chinese J.Lasers,2011,38(1):0105004

[7] Wu Jun, Chen Weimin, Zhang Yaling et al..Affect mechanism and experimental research on performance degeneration of FBG under the action of alternate temperature test $[\mathrm{J}]$. J. Optovlvctr.Laser,2010,21(9):1301-1305

[8] P. S. Andre, A. L. Teixeira, M. J. N. Lima et al.. Single fiber Bragg grating degenerated into optical cavity clock recovers for clock recovery purposes[C].Proceeding of IEEE,2005.341-344

[9] F.Iacoviello, M.Boniardi, G.M.La Vecchia. Fatigue crack propagation in austen-ferritic duplex stainless steel 22Cr5Ni [J]. International Journal of Fatigue, 1999, (21): 957-963

[10] A.K.Vasudevan, K.Sandanda, K.Rajan. Role of microstructure on the growth of long fatigue cracks[J]. Int. J. Fatigue, 1997, 19(18-19): 641-646. 